\title{
Permuting Tri-Derivations of Semiprime Gamma Rings
}

\author{
K. K. Dey ${ }^{*}$ and A. C. Paul \\ Department of Mathematics, Rajshahi University, Rajshahi-6205, Bangladesh
}

Received 11 July 2012, accepted in final revised form 4 October 2012

\begin{abstract}
We study some properties of permuting tri-derivations on semiprime $\Gamma$-rings with a certain assumption. Let $M$ be a 3-torsion free semiprime $\Gamma$-ring satisfying a certain assumption and let $I$ be a non-zero ideal of $M$. Suppose that there exists a permuting tri-derivation $D$ : $M \times M \times M \rightarrow M$ such that $d$ is an automorphism commuting on $I$ and also $d$ is a trace of $D$. Then we prove that $I$ is a nonzero commutative ideal. Various characterizations of $M$ are obtained by means of tri-derivations.
\end{abstract}

Keywords: Tri-derivation; Semiprime $\Gamma$-ring; Commutative ideal; Commuting map; Permuting map.

(C) 2013 JSR Publications. ISSN: 2070-0237 (Print); 2070-0245 (Online). All rights reserved. doi: http://dx.doi.org/10.3329/jsr.v5i1.9549 J. Sci. Res. 5 (1), 55-66 (2013)

\section{Preliminaries}

Gamma rings were first introduced by Nabusawa [1] and then Barnes [2] generalized the definition of $\Gamma$-rings. In this paper we work on $\Gamma$-rings due to Barnes [2]. Throughout this paper, $M$ will represent a $\Gamma$-ring and $Z(M)$ will be its center. A $\Gamma$-ring $M$ is prime if $x \Gamma M \Gamma y=0$ implies that $x=0$ or $y=0$, and is semiprime if $x \Gamma M \Gamma x=0$ implies $x=0$. Let $x, y \in M, \alpha \in \Gamma$, the commutator $x \alpha y-y \alpha x$ will be denoted by $[x, y]_{\alpha}$. We know that $[x \beta y$, $z]_{\alpha}=x \beta[y, z]_{\alpha}+[x, z]_{\alpha} \beta y+x[\beta, \alpha]_{z} y$ and $[x, y \beta z]_{\alpha}=y \beta[x, z]_{\alpha}+[x, y]_{\alpha} \beta z+y[\beta, \alpha]_{x} z$ for all $x, y, z \in M, \alpha, \beta \in \Gamma$. We shall take an assumption (*) $x \alpha y \beta z=x \beta y \alpha z$ for all $x, y, z \in M, \alpha$, $\beta \in \Gamma$. Using the assumption (*) the above identities reduce to $[x \beta y, z]_{\alpha}=x \beta[y, z]_{\alpha}+[x$, $z]_{\alpha} \beta y$ and $[x, y \beta z]_{\alpha}=y \beta[x, z]_{\alpha}+[x, y]_{\alpha} \beta z$, for all $x, y, z \in M$ and for all $\alpha, \beta \in \Gamma$ which are used extensively in our results.

Let $I$ be a nonempty subset of $M$. Then a map $d: M \rightarrow M$ is said to be commuting (resp. centralizing) on $I$ if $[d(x), x]_{\alpha}=0$ for all $x \in I, \alpha \in \Gamma$ (resp. $[d(x), x]_{\alpha} \in Z(M)$ for all $x \in I$,

\footnotetext{
*Corresponding author: kkdmath@yahoo.com
} 
$\alpha \in \Gamma)$, and is called central if $d(x) \in Z(M)$ for all $x \in M, \alpha \in \Gamma$. Every central mapping is obviously commuting but not conversely in general, and $d$ is called skew-centralizing on a subset $I$ of $M$ (resp. skew-commuting on a subset $I$ of $M$ ) if $d(x) \alpha x+x \alpha d(x) \in Z(M)$ holds for all $x \in I, \alpha \in \Gamma$ (resp. $d(x) \alpha x+x \alpha d(x)=0$ holds for all $x \in I, \alpha \in \Gamma)$. Recall that $M$ is said to be $n$-torsion free, where $n \neq 0$ is an integer, if whenever $n x=0$, with $x \in M$ then $x=0$. An additive map $d: M \rightarrow M$ is called a derivation if $d(x \alpha y)=d(x) \alpha y+x \alpha d(y)$ for all $x$, $y \in M, \alpha \in \Gamma$. By a bi-derivation we mean a bi-additive map $D: M \times M \rightarrow M$ (i.e., $D$ is additive in both arguments), which satisfies the relations $D(x \alpha y, z)=D(x, z) \alpha y+x \alpha D(y$, $z$ ) and $D(x, y \alpha z)=D(x, y) \alpha z+y \alpha D(x, z)$ for $x, y \in M, \alpha \in \Gamma$. Let $D$ be symmetric, that is $D(x, y)=D(y, x)$ for the $x, y \in M$. The map $d: M \rightarrow M$ defined by $d(x)=D(x, x)$ for all $x \in M$ is called the trace of $D$. A map $D: M \times M \times M \rightarrow M$ will be said to be permuting if the equation $D(x, y, z)=D(x, z, y)=D(z, x, y)=D(y, z, x)=D(z, y, x)$ for all $x, y, z \in M$. A map $d: M \rightarrow M$ defined by $d(x)=D(x, x, x)$ for all $x \in M$, where $D: M \times M \times M \rightarrow M$ is a permuting map is called the trace of $D$. It is obvious that, in case when $D: M \times M \times M \rightarrow$ $M$ is a permuting map which is also tri-additive (i.e., additive in each argument), the trace $d$ of $D$ satisfies the relation $d(x+y)=d(x)+d(y)+3 D(x, x, y)+3 D(x, y, y)$ for all $x, y \in M$. Since we have $D(0, y, z)=D(0+0, y, z)=D(0, y, z)+D(0, y, z)$ for all $y, z \in M$, we obtain $D(0, y, z)=0$ for all $y, z \in M$. Hence we get $D(0, y, z)=D(x-x, y, z)=D(x, y, z)+D(-x, y$, $z)=0$ and so we see that $D(-x, y, z)=-D(x, y, z)$ for all $x, y, z \in M$. This implies that $d$ is an odd function. A tri-additive map $D: M \times M \times M \rightarrow M$ will be called a tri-derivation if the relations $D(x \alpha w, y, z)=D(x, y, z) \alpha w+x \alpha D(w, y, z), D(x, y \alpha w, z)=D(x, y, z) \alpha w+$ $y \alpha D(x, w, z)$ and $D(x, y, z \alpha w)=D(x, y, z) \alpha w+z \alpha D(x, y, w)$ are fulfilled for all $x, y, z$, $w \in M, \alpha \in \Gamma$. If $D$ is permuting, then the above three relations are equivalent to each other.

Let $M$ be commutative $\Gamma$-ring. A map $D: M \times M \times M \rightarrow M$ defined by $(x, y, z) \rightarrow$ $d(x) \alpha d(y) \beta d(z)$ for all $x, y, z \in M, \alpha, \beta \in \Gamma$, is a tri-derivation where $d$ is a derivation on $M$.

Ozturk et al. [3] studied on symmetric bi-derivations on prime $\Gamma$-rings. Some fruitful results of prime $\Gamma$-rings were obtained by these authors. Ozturk [4] obtained some properties concerning to the mapping permuting tri-derivations on prime and semiprime $\Gamma$-rings. Permuting tri-derivations in prime and semiprime $\Gamma$-rings had been studied by Ozden et al. [5]. Some remarkable results of these $\Gamma$-rings were obtained by them. An example of a permuting tri-derivation has also been given by these authors [5].

In this paper, we study and investigate some results concerning a permuting triderivation $D$ on non-commutative 3-torsion free semiprime $\Gamma$-rings $M$. Some characterizations of semiprime $\Gamma$-rings are obtained by means of permuting triderivations.

First we prove the following lemmas which will be needed in our results. 


\section{Lemma 1.1}

Let $M$ be a semiprime $\Gamma$-ring. Then $M$ contains no nonzero nilpotent ideal.

\section{Proof.}

Let $I$ be a nilpotent ideal of $M$. Then $(I \Gamma)^{n} I=0$ for some positive integer $n$. Let us assume that $n$ is minimum. Now suppose that $n \geq 1$. Since $I \Gamma M \subset I$, we then have $(I \Gamma)^{n-}$ ${ }^{1} I \Gamma M \Gamma(I \Gamma)^{n-1} I \subset(I \Gamma)^{\mathrm{n}-1} I(I \Gamma)^{\mathrm{n}} I=(I \Gamma)^{n} I(I \Gamma)^{n-2} I=0$. Hence by the semiprimeness of $M$ we get $(I \Gamma)^{n-1} I=0$, a contradiction to the minimality of $n$. Therefore $n=1$. Thus $I \Gamma I=0$. Then $I \Gamma M \Gamma I \subset I \Gamma I=0$. Since $M$ is semiprime, it gives $I=0$. This completes the proof.

The above lemma gives us the following corollary.

\section{Corollary 1.2}

Every prime $\Gamma$-ring has no nilpotent ideals.

\section{Lemma 1.3 [15 Theorem 4.1]}

Let $M$ be a 2, 3-torsion free prime $\Gamma$-ring. Let $D(.$, ., .) be permuting tri-derivation of $M$ with the trace $d$. If

$$
\operatorname{a\alpha d}(x)=0, x \in M, \alpha \in \Gamma
$$

where $a$ is a fixed element of $M$, then either $a=0$ or $D=0$.

\section{Lemma 1.4}

Let $M$ be a 2-torsion free semiprime $\Gamma$-ring. If $x \alpha x=0$ then $x \in Z(M)$ for all $x \in M, \alpha \in \Gamma$.

\section{Proof:}

We have $x \alpha x=0$ for all $x \in M, \alpha \in \Gamma$. Replacing $x$ by $x+y$, we get $x \alpha y+y \alpha x=0$ for all $x$, $y \in M, \alpha \in \Gamma$.

Right-multiplying by $\beta x$ we obtain $x \alpha y \beta x=0$ for all $x, y \in M, \alpha, \beta \in \Gamma$. Replacing $y$ by $y \gamma z$ and right-multiplying by $\alpha y$ we get $x \alpha y \gamma z \beta x \alpha y=0$ for all $x, y, z \in M, \alpha, \beta, \gamma \in \Gamma$. Since $M$ is semiprime $\Gamma$-ring, we obtain $x \alpha y=0$ for all $x, y \in M, \alpha \in \Gamma$. By the same method, we get $y \alpha x$ $=0$ for all $x, y \in M, \alpha \in \Gamma$. By subtracting those, we obtain $[x, y]_{\alpha}=0$, for all $x, y \in M, \alpha \in \Gamma$, then $x \in Z(M)$ for all $x \in M$.

\section{Lemma 1.5}

Let $M$ be a semiprime $\Gamma$-ring satisfying the condition (*). If $x \alpha x \in Z(M)$ then $x \in Z(M)$ for all $x \in M, \alpha \in \Gamma$.

\section{Proof:}

We have $x \alpha x \in Z(M)$ for all $x \in M, \alpha \in \Gamma$. Then $[x \alpha x, z]_{\beta}=0$ for all $z \in M, \alpha, \beta \in \Gamma$. Replacing $x$ by $x+y$, we get $[x \alpha y+y \alpha x, z]_{\beta}=0$ for all $x, y, z \in M, \alpha, \beta \in \Gamma$. Since $y \beta z \alpha x=y \alpha z \beta x$, we have $x \alpha[y, z]_{\beta}+[x, z]_{\beta} \alpha y+[y \alpha x, z]_{\beta}=0$ for all $x, y, z \in M, \alpha, \beta \in \Gamma$. 
Similarly, $[y, z]_{\beta} \alpha x+y \alpha[x, z]_{\beta}+[x \alpha y, z]_{\beta}=0$ for all $x, y, z \in M, \alpha, \beta \in \Gamma$.

Using the relation $[x \alpha y+y \alpha x, z]_{\beta}=0$ and replacing $y$ by $x \alpha x$ we obtain $[x, z]_{\beta} \alpha x \alpha x=0$ for all $x, z \in M, \alpha, \beta \in \Gamma$.

Left-multiplying by $x \alpha$ and right-multiplying $\alpha[x, z]_{\beta} \alpha x$, we get $\left(x \alpha[x, z]_{\beta} \alpha x\right) \alpha(x \alpha[x$, $\left.z]_{\beta} \alpha x\right)=0$ for all $x, z \in M, \alpha, \beta \in \Gamma$. We obtain $x \alpha[x, z]_{\beta} \alpha x=0$ for all $x, z \in M, \alpha, \beta \in \Gamma$. Leftmultiplying by $[x, z]_{\beta} \alpha$ with using Lemma 1.4 , we obtain $[x, z]_{\beta} \alpha x=0$ for all $x, z \in M, \alpha$, $\beta \in \Gamma$. Right-multiplying by $\delta z$, we get $[x, z]_{\beta} \alpha x \delta z=0$ for all $x, z \in M, \alpha, \beta, \delta \in \Gamma$. Again using the relation $[x, z]_{\beta} \alpha x=0$ and replacing $z$ by $z \delta z$, we obtain $[x, z]_{\beta} \alpha z \delta x=0$ for all $x$, $z \in M, \alpha, \beta, \delta \in \Gamma$. Subtracting we obtain $x \in Z(M)$ for all $x \in M$.

\section{Lemma 1.6}

Let $M$ be a 3-torsion free prime $\Gamma$-ring satisfying the condition (*) and let $I$ be a non zero ideal of $M$. If there exists a permuting tri-derivation $D: M \times M \times M \rightarrow M$ such that $\mathrm{d}$ is commuting on $I$, where $d$ is the trace of $D$, then we have $D=0$.

\section{Proof.}

Suppose that

$$
[d(x), x]_{\beta}=0 \text { for all } x \in I, \beta \in \Gamma
$$

Linearizing (2) we get,

$$
\begin{aligned}
& {[d(x), y]_{\beta}+[d(y), x]_{\beta}+3[D(x, x, y), x]_{\beta}+3[D(x, y, y), x]_{\beta}+3[D(x, x, y), y]_{\beta}+} \\
& 3[D(x, y, y), y]_{\beta}=0 \text { for all } x, y \in I, \beta \in \Gamma
\end{aligned}
$$

Putting $-\mathrm{x}$ instead of $\mathrm{x}$ in (3) and since $d$ is odd, we obtain

$$
[D(x, x, y), x]_{\beta}=0 \text { for all } x, y \in I, \beta \in \Gamma
$$

Putting $x=x+y$ in (4) and then we obtain

$$
[d(y), x]_{\beta}+3[D(x, y, y), x]_{\beta}=0 \text { for all } x, y \in I, \beta \in \Gamma
$$

Replacing $y \alpha x$ for $x$ in (3) we get

$$
[d(y), y \alpha x]_{\beta}+3[D(y \alpha x, x, y), y]_{\beta}=y \alpha[d(y), x]_{\beta}+3 d(y) \alpha[x, y]_{\beta}+3 y \alpha[D(x, y, y), y]_{\beta}=0
$$

for all $x, y \in I, \alpha, \beta \in \Gamma$, which implies that

$$
y \alpha\left([d(y), x]_{\beta}+3[D(x, y, y), y]_{\beta}\right)+3 d(y) \alpha[x, y]_{\beta}=0
$$

By using (5) we have $d(y) \alpha[x, y]_{\beta}=0$ for all $x, y \in I, \alpha, \beta \in \Gamma$ on account of (5). Since $I$ is a nonzero non-commutative prime $\Gamma$-ring, it follows from (3) and Lemma 1.3 that, for all $y \in I$ with $y \notin Z(M)$, we have $d(y)=0$ since for every fixed $y \in I$, a map $x \rightarrow[x, y]_{\beta}$ is a derivation on $I$. 
Now, let $x \in I$ with $x \in Z(M)$ and $y \in I$ with $y \notin Z(M)$. Then $x+y \notin Z(M)$ and $-y \notin Z(M)$. Thus we have

$d(x+y)=d(x)+3 D(x, x, y)+3 D(x, y, y)=0$ which shows that $d(x-y)=d(x)-3 D(x$, $x, y)+3 D(x, y, y)=0$ which shows that

$$
d(x)+3 D(x, y, y)=0
$$

Replacing $y \in I(y \notin Z(M))$ by $2 y$ in (7) we obtain that $D(x, y, y)=0$ and so the relation (7) gives $d(x)=0$ for all $x \in I$ with $x \in Z$. Therefore we obtain $d(x)=0$ for all $x \in I$.

On the other hand, since the relation $D(x, x, y)+D(x, y, y)=0$ fulfilled for all $x, y \in I$, it follows that

$$
D(x, x, y)+D(x, y, y)=0 \text { for all } x, y \in I,
$$

and substituting $y+z$ for $y$ in (8) we obtain that $2 D(x, y, z)=0=D(x, y, z)$ for all $x, y \in I$.

Let us substitute $w \alpha x(w \in M)$ for $x$ in the above relation $D(x, y, z)=0$ for all $x, y, z \in I$. Then we have

$D(w, y, z) \alpha x=0$. Hence $D(w, y, z) \alpha x \beta D(w, y, z)=0$. Since $M$ is prime, we get $D(w, y$, $z)=0$ for all $y, z \in I, w \in M$. Also, substituting $y \delta v(v \in M)$ for $y$ in this relation, we have $y \delta D(w, v, z)=0$ and so $D(w, v, z) \beta y \delta D(w, v, z)=0$. Again, by primeness of $M$, we obtain that $D(w, v, z)=0$ for all $z \in I, w, v \in M$. Furthermore, replacing $z$ by $u \gamma z(u \in M, \gamma \in \Gamma)$ in the relation $D(w, v, z)=0$, we get $D(w, v, u)=0$. The primeness of $M$ implies that $D(w, v, u)=$ 0 for all $u, v, w \in M$.

\section{Lemma 1.7}

Let $M$ be a 3-torsion free semiprime $\Gamma$-ring satisfying the condition (*) and $I$ be a nonzero ideal of $M$. If there exists a permuting tri-derivation $D: M \times M \times M \rightarrow M$ such that $\mathrm{d}$ is centralizing on $I$, where $d$ is the trace of $D$, then $d$ is commuting on $I$.

\section{Proof:}

Assume that

$[d(x), x]_{\beta} \in Z(M)$ for all $x \in I, \beta \in \Gamma$

By linearizing (9) we get,

$[d(x), y]_{\beta}+[d(y), x]_{\beta}+3[D(x, x, y), x]_{\beta}+3[D(x, y, y), x]_{\beta}+3[D(x, x, y), y]_{\beta}$

$+3[D(x, y, y), y]_{\beta} \in Z(M)$, for all $x, y \in I, \beta \in \Gamma$.

We substitute $-x$ for $x$ in (10) we get

$[D(x, y, y), x]_{\beta}+[D(x, x, y), y]_{\beta} \in Z(M)$, for all $x, y \in I, \beta \in \Gamma$

Replacing $x$ by $x+y$ in (11) we have

$[d(y), x]_{\beta}+3[D(x, y, y), y]_{\beta} \in Z(M)$, for all $x, y \in I, \beta \in \Gamma$ 
Taking $x=y \delta y$ in (12) and invoking (9) show that

$[d(y), y \delta y]_{\beta}+3[D(y \delta y, y, y), y]_{\beta}=8[d(y), y]_{\beta} \delta y \in Z(M)$, for all $y \in I, \beta, \delta \in \Gamma$

Commuting with $d(\mathrm{y})$ in (13) gives

$8[d(y), y]_{\beta} \delta[d(y), y]_{\beta}=0$, for all $y \in I, \beta, \delta \in \Gamma$

On the other hand, substituting $x$ for $y \gamma x$ in (14)

$[d(y), y \gamma x]_{\beta}+3[D(y \gamma x, x, y), y]_{\beta}=y \gamma[d(y), x]_{\beta}+3 d(y) \gamma[x, y]_{\beta}+3[D(x, y, y), y]_{\beta}$

$+4[d(y), y]_{\beta} \gamma x \in Z(M)$ for all $x, y \in I, \beta, \gamma \in \Gamma$

Hence we have

$\left[y \gamma\left\{[d(y), x]_{\beta}+3[D(x, y, y), y]_{\beta} \gamma[x, y]_{\beta}\right\}, y\right]_{\beta}+\left[3 d(y) \gamma[x, y]_{\beta}+4[d(y), y]_{\beta} \gamma x, y\right]_{\beta}=0$

for all $x, y \in I, \beta, \gamma \in \Gamma$

So we get $\left[3 d(y) \gamma[x, y]_{\beta}, y\right]_{\beta}+7[d(y), y]_{\beta} \gamma[x, y]_{\beta}=0$, for all $x, y \in I, \beta, \gamma \in \Gamma$, according to (14).

Substituting $d(y) \lambda x$ for $x$ in (15), it follows that

$d(y) \gamma\left\{3 d(y) \lambda\left[[x, y]_{\beta}, y\right]_{\beta}+7[d(y), y]_{\beta} \gamma[x, y]_{\beta}\right\}+6 d(y) \gamma[d(y), y]_{\beta} \gamma[x, y]_{\beta}$

$+7[d(y), y]_{\beta} \gamma[d(y), y]_{\beta} \gamma x$, for all $x, y \in I, \beta, \gamma \in \Gamma$,

which by (16) implies

$6 d(y) \gamma[d(y), y]_{\beta} \gamma[x, y]_{\beta}+7[d(y), y]_{\beta} \gamma[d(y), y]_{\beta} \gamma x=0$ for all $x, y \in I, \beta, \gamma \in \Gamma$

Letting $x=[d(y), y]_{\beta}$ in (18) we arrive at $[d(y), y]_{\beta} \gamma[d(y), y]_{\beta} \gamma[d(y), y]_{\beta}=0$ and so we get

$7[d(y), y]_{\beta} \gamma[d(y), y]_{\beta} \gamma 7[d(y), y]_{\beta} \gamma[d(y), y]_{\beta}=0$

Since $M$ is a semiprime $\Gamma$-ring $7[d(y), y]_{\beta} \gamma[d(y), y]_{\beta}=0$ for all $x, y \in I, \beta, \gamma \in \Gamma$. Hence, the relations (16) and (19) yield $[d(y), y]_{\beta} \gamma[d(y), y]_{\beta}=0$ for all $y \in I, \beta, \gamma \in \Gamma$. Since the center of a semiprime ring contains no nonzero nilpotent elements, we conclude that $[d(y)$, $y]_{\beta}=0$ for all $y \in I, \beta \in \Gamma$. This completes the proof.

\section{Lemma 1.8}

Let $M$ be a 3-torsion free prime $\Gamma$-ring and let $I$ be a nonzero ideal of $M$. If there exists a nonzero permuting tri-derivation $D: M \times M \times M \rightarrow M$ such that $\mathrm{d}$ is centralizing on $I$, where $\mathrm{d}$ is the trace of $D$, then $M$ is commutative.

\section{Proof:}

Suppose that $M$ is non-commutative. Then it follows from Lemma 1.3 that is commuting on $I$. Hence Lemma 1.6 gives $D=0$ which proves the Lemma. 


\section{Lemma 1.9}

Let $M$ be a semiprime $\Gamma$-ring satisfying the condition (*). If there exists $a \in M$ such that $a \alpha[x, y]_{\beta}=0$ holds for all pairs $x, y \in M, \alpha, \beta \in \Gamma$. In this case, $a \in Z(M)$.

\section{Proof:}

We have $[z, a]_{\beta} \alpha x \delta[z, a]_{\beta}=z \beta a \alpha x \delta[z, a]_{\beta}-a \beta z \alpha x \delta[z, a]_{\beta}=z \beta a \alpha[z, x \delta a]_{\beta}-z \beta a \alpha[z, x]_{\beta} \delta a$ $-a \beta[z, z \alpha x \delta a]_{\beta}+a \beta[z, z \alpha x]_{\beta} \delta a=0$.

Hence $a \in Z(M)$. Since $z \alpha a \delta w \gamma[x, y]_{\beta}=0$ for all $z, w, x, y \in M, \alpha, \beta, \delta, \gamma \in \Gamma$, we can repeat the above argument with $z \alpha a \gamma w$ instead of a to obtain $M \Gamma a \Gamma M \in Z(M)$ and now it is obvious that the ideal generated by $a$ is central.

\section{Permuting Tri-Derivations}

We prove some results on permuting tri-derivations.

\section{Theorem 2.1}

Let $M$ be a 3-torsion free semiprime $\Gamma$-ring satisfying the condition (*) and let $I$ be a nonzero ideal of $M$. If there exists a permuting tri-derivation $D: M \times M \times M \rightarrow M$ such that $\mathrm{d}$ is an automorphism commuting on $\mathrm{I}$, where $d$ is the trace of $D$, then $I$ is a nonzero commutative ideal.

\section{Proof:}

Suppose that

$[d(x), x]_{\beta}=0$ for all $x \in I, \beta \in \Gamma$.

Substituting $x$ by $x+y$ leads to

$[d(x), y]_{\beta}+[d(y), x]_{\beta}+3[D(x, x, y), x]_{\beta}+3[D(x, y, y), x]_{\beta}+3[D(x, x, y), y]_{\beta}$

$+3[D(x, y, y), y]_{\beta}=0$ for all $x, y \in I, \beta \in \Gamma$

Putting $-x$ instead of $x$ in (21) we get

$[D(x, y, y), x]_{\beta}+[D(x, x, y), y]_{\beta}=0$ for all $x, y \in I, \beta \in \Gamma$.

Since $d$ is odd, we set $x=x+y$ in (22) and then use (20) and (22) to obtain

$[d(y), x]_{\beta}+3[D(x, y, y), y]_{\beta}=0$ for all $x, y \in I, \beta \in \Gamma$.

Let us write $y \alpha x$ instead of $x$ in (23), we obtain

$[d(y), y \alpha x]_{\beta}+3[D(y \alpha x, y, y), y]_{\beta}=y \alpha[d(y), x]_{\beta}+3 d(y) \alpha[x, y]_{\beta}+3 y \alpha[D(x, y, y), y]_{\beta}=$ $y \alpha\left([d(y), x]_{\beta}+3[D(x, y, y), y]_{\beta}\right)+3 d(y) \alpha[x, y]_{\beta}=0$ for all $x, y \in I, \alpha, \beta \in \Gamma$. Then $d(y) \alpha[x$, $y]_{\beta}=0$ for all $x, y \in I, \alpha, \beta \in \Gamma$, since $d$ is an automorphism, we obtain $y \alpha[x, y]_{\beta}=0$ for all $x$, $y \in I, \alpha, \beta \in \Gamma$. Replacing $x$ by $y \alpha x$, we get 
$y \alpha x \gamma[x, y]_{\beta}=0$ for all $x, y \in I, \alpha, \beta, \gamma \in \Gamma$.

Again left-multiplying by $x$ implies that

$x \alpha y \gamma[x, y]_{\beta}=0$ for all $x, y \in I, \alpha, \beta, \gamma \in \Gamma$.

Subtracting (24) and (25) with using $M$ is semiprime $\Gamma$-ring, we completes our proof. By same method in Theorem 2.1, it is easy to proof the following results.

\section{Corollary 2.2}

Let $M$ be a 3-torsion free semiprime $\Gamma$-ring satisfying the condition (*) and $I$ be an ideal of $M$. If there exists a permuting tri-derivation $D: M \times M \times M \rightarrow M$ such that $d$ is commutating on $I$, where $d$ is the trace of $D$, then $I$ is a central ideal.

\section{Theorem 2.3}

Let $M$ be a 3-torsion free semiprime $\Gamma$-ring satisfying the condition (*). If there exist a permuting tri-derivation $D: M \times M \times M \rightarrow M$ such that $\mathrm{d}$ is an automorphism commuting on $M$, where $d$ is the trace of $D$, then $M$ is commutative.

\section{Theorem 2.4}

Let $M$ be a 6-torsion free semiprime $\Gamma$-ring satisfying the condition (*). If there exists a permuting tri-derivation $D: M \times M \times M \rightarrow M$ such that $d$ is an automorphism centralizing on $M$, where $d$ is the trace of $D$, then $M$ is commutative.

\section{Proof:}

Assume that

$[d(x), x]_{\beta} \in Z(M)$ for all $x \in M$ and $\beta \in \Gamma$.

Replacing $x$ by $x+y$ and again using (26), we obtain

$[d(x), y]_{\beta}+[d(y), x]_{\beta}+3[D(x, x, y), x]_{\beta}+3[D(x, y, y), x]_{\beta}+3[D(x, x, y), y]_{\beta}$

$+3[D(x, y, y), y]_{\beta} \in Z(M)$ for all $x, y \in M, \beta \in \Gamma$.

Replacing $x$ by $-x$ in (27) we get

$[D(x, y, y), x]_{\beta}+[D(x, x, y), y]_{\beta} \in Z(M)$ for all $x, y \in M, \beta \in \Gamma$.

Replacing $x$ by $x+y$ in (28), we obtain

$[d(y), x]_{\beta}+3[D(x, y, y), y]_{\beta} \in Z(M)$ for all $x, y \in M, \beta \in \Gamma$.

Taking $x=y \alpha y$ in (29) and invoking (26), we get

$[d(y), y \alpha y]_{\beta}+3[D(y \alpha y, y, y), y]_{\beta}=8[d(y), y]_{\beta} \alpha y \in Z(M)$ for all $y \in M, \alpha, \beta \in \Gamma$.

Now commuting (30) with $d(y)$ yields 
$8[d(y), y]_{\beta} \alpha[d(y), y]_{\beta}=0$ for all $y \in M, \alpha, \beta \in \Gamma$.

Again substituting $x$ by $y \alpha x$ in (29) gives

$[d(y), y \alpha x]_{\beta}+3[D(y \alpha x, y, y), y]_{\beta}=y \alpha\left([d(y), x]_{\beta}+3[D(x, y, y), y]_{\beta}\right)+3 d(y) \alpha[x, y]_{\beta}+$ $4[d(y), y]_{\beta} \alpha x \in Z(M)$ for all $x, y \in M, \alpha, \beta \in \Gamma$. Then $\left[y \alpha\left([d(y), x]_{\beta}+3[D(x, y, y), y]_{\beta}\right), y\right]_{\beta}+$ $\left[3 d(y) \alpha[x, y]_{\beta}+4[d(y), x]_{\beta} \alpha x, y\right]_{\beta}=0$ for all $x, y \in M, \alpha, \beta \in \Gamma$. And so we get

$3 d(y) \alpha\left[[x, y]_{\beta}, y\right]_{\beta}+7[d(y), y]_{\beta} \alpha[x, y]_{\beta}=0$ for all $x, y \in M, \alpha, \beta \in \Gamma$.

Since d acts as an automorphism with $M$ is 6-torsion free the relation (31) reduces to $y \alpha\left[[x, y]_{\beta}, y\right]_{\beta}=0$ for all $x, y \in M, \alpha, \beta \in \Gamma$.

Replacing $x$ by $r \delta x$, we get

$y \alpha x \delta\left[[x, y]_{\beta}, y\right]_{\beta}+2 y \alpha[x, y]_{\beta}=0$ for all $x, y \in M, \alpha, \beta, \delta \in \Gamma$.

Replacing $y$ by $-y$ in (32) and subtracting with (32), gives

$4 y \delta[x, y]_{\beta}=0$ for all $x, y \in M, \beta, \delta \in \Gamma$.

Replacing $x$ by $x \gamma r$ and left-multiplying by $s$, we obtain

$4 y \delta x \alpha[r, y]_{\beta}=0$ for all $x, y, r, s \in M, \alpha, \beta, \delta \in \Gamma$.

Again in (33) replacing $\mathrm{x}$ by $\mathrm{x} \lambda \mathrm{m}$ and $\mathrm{x}$ by $\mathrm{s} \delta \mathrm{x}$, we get

$4 y \gamma_{s} \delta x \alpha[m, y]_{\beta}=0$ for all $x, y, m, s \in M, \alpha, \beta, \delta, \gamma \in \Gamma$.

Subtracting (34) and (35) with using $M$ is 6-torsion free semiprime, we obtain $[s, y]_{\beta}=$ 0 for all $s, y \in M$. Thus, we get $M$ is commutative.

\section{Theorem 2.5}

Let $M$ be a 3-torsion free semiprime $\Gamma$-ring satisfying the condition (*). If there exists a permuting tri-derivation $D: M \times M \times M \rightarrow M$ such that $d$ is commuting on $M$, where $d$ is the trace of $D$, then $d$ is a central mapping.

\section{Proof:}

We have

$[d(x), x]_{\beta}=0$ for all $x \in M, \beta \in \Gamma$.

The substitution of $x$ in (36) by $x+y$ leads to

$[d(x), y]_{\beta}+[d(y), x]_{\beta}+3[D(x, x, y), x]_{\beta}+3[D(x, y, y), x]_{\beta}+3[D(x, x, y), y]_{\beta}$

$+3[D(x, y, y), y]_{\beta}=0$ for all $x, y \in M, \beta \in \Gamma$.

Putting $-x$ instead of $x$ in (37) we obtain,

$[D(x, y, y), x]_{\beta}+[D(x, x, y), y]_{\beta}=0$ for all $x, y \in M, \beta \in \Gamma$. 
Since $\mathrm{d}$ is odd, we set $x=x+y$ in (38) with using (36) and (37), we get

$[d(y), x]_{\beta}+3[D(x, y, y), y]_{\beta}=0$ for all $x, y \in M, \beta \in \Gamma$.

Let us write in (39) $y \alpha x$ instead of $x$, we obtain according to (39) and since $M$ is 3torsion semiprime $d(y) \alpha[x, y]_{\beta}=0$ for all $x, y \in M, \beta \in \Gamma$.

Applying Lemma 1.9, the above relation gives $d(y) \in Z(M)$ for all $y \in M$, thus we completes the proof of the theorem.

\section{Theorem 2.6}

Let $M$ be a 3-torsion free semiprime $\Gamma$-ring. If there exists a permuting tri-derivation $D: M$ $\times M \times M \rightarrow M$ such that $d$ is commuting on $M$, where $d$ is the trace of $D$, then $D$ is commuting (resp. centralizing).

\section{Proof:}

We can restrict our attention to the relation

$$
[d(x), x]_{\beta}=0 \text { for all } x \in M, \beta \in \Gamma .
$$

The substitution of $x+y$ for $x$ in above relation gives

$$
\begin{aligned}
& {[d(x), y]_{\beta}+[d(y), x]_{\beta}+3[D(x, x, y), x]_{\beta}+3[D(x, y, y), x]_{\beta}+3[D(x, x, y), y]_{\beta}} \\
& +3[D(x, y, y), y]_{\beta}=0 \text { for all } x, y \in M, \beta \in \Gamma
\end{aligned}
$$

Now, by the same method in Theorem 2.5 , we arrive at

$$
y \delta[d(y), x]_{\beta}+3 d(y) \delta[x, y]_{\beta}+3 y \delta[D(x, y, y), y]_{\beta}=0 \text { for all } x, y \in M, \beta, \delta \in \Gamma .
$$

which implies that

$$
d(y) \delta[x, y]_{\beta}=0 \text { for all } x, y \in M, \beta, \delta \in \Gamma .
$$

Applying Lemma 1.5, the above relation gives $d(y) \in Z(M)$ for all $x \in M$. By substitution the relation $d(y) \in Z(M)$ in (41) with using replacing $x$ by $y$ and $M$ is 3-torsion free semiprime, we obtain

$$
[D(y, y, y), y]_{\beta}=0 \text { for all } x, y \in M, \beta \in \Gamma
$$

Then $D$ is commuting (resp. centralizing) of $M$.

\section{Theorem 2.7}

Let $M$ be a non-commutative 3-torsion free semiprime $\Gamma$-ring satisfying the condition (*). If there exists a permuting tri-derivation $D: M \times M \times M \rightarrow M$ such that d is skewcommuting on $M$, where $d$ is the trace of $D$, then $d$ is commuting.

\section{Proof:}

We have $d(x) \alpha x+x \alpha d(x)=0$ for all $x \in M$. Replacing $x$ by $x+y$, we obtain 


$$
\begin{aligned}
& d(y) \alpha x+3 D(x, x, y) \alpha x+3 D(x, y, y) \alpha x+d(x) \alpha y+3 D(x, x, y) \alpha y+3 D(x, y, y) \alpha y \\
& +x \alpha d(y)+3 x \alpha D(x, x, y)+3 x \alpha D(x, y, y)+y \alpha d(y)+3 y \alpha D(x, x, y) \\
& +3 y \alpha D(x, y, y)=0 \text { for all } x, y \in M, \alpha \in \Gamma
\end{aligned}
$$

We substitute $-x$ for $x$ in (45) we get $3 D(x, y, y) \alpha x+3 D(x, x, y) \alpha y+3 x \alpha D(x, y, y)+$ $3 y \alpha D(x, x, y)=0$ for all $x, y \in M, \alpha \in \Gamma$.

Since $M$ is 3-torsion free, we obtain

$$
D(x, x, y) \alpha x+D(x, x, y) \alpha y+x \alpha D(x, y, y)+y \alpha D(x, x, y)=0 \text { for all } x, y \in M, \alpha \in \Gamma
$$

Again we substituting $x \beta y$ for $x$ in (46) then we get

$$
\begin{aligned}
& x \alpha D(y, y, y) \beta y+D(x, y, y) \alpha x \beta y+x \alpha y \beta D(y, y, y)+D(x, y, y) \alpha y=0 \\
& \text { for all } x, y \in M, \alpha, \beta \in \Gamma
\end{aligned}
$$

We substitute $-x$ for $x$ in (47) and compare (47) with the result to get $D(x, y, y) \alpha x \beta y=$ 0 for all $x, y \in M$. Replacing $x$ by $y$ and since $d$ is the trace of $D$, we obtain $d(y) \alpha y \beta y=0$ for all $y \in M$. Left- multiplying by $y$ and right-multiplying by $d(y) \delta y$ with using Lemma 1.4 , we obtain

$$
y \delta d(y) \beta y=0 \text { for all } y \in M, \beta, \delta \in \Gamma .
$$

Left- multiplying (48) by $d(y)$ with using Lemmas (1.1 and 1.3) gives

$$
d(y) \beta y=0 \text { for all } y \in M, \beta \in \Gamma \text {. }
$$

Right- multiplying (48) by $d(y)$ with using Lemmas (1.1 and 1.3) and subtracting the result with (49), we obtain $[d(y), y]_{\beta}=0$ for all $y \in M, \beta \in \Gamma$.

By Theorem 2.3, we complete our proof.

By the same method in Theorem 2.8, with using Lemmas (1.4 and 1.5), it is easy to proof the following corollary.

\section{Theorem 2.8}

Let $M$ be a non-commutative 3-torsion free semiprime $\Gamma$-ring satisfying the condition (*) and $I$ be a non-zero ideal of $M$. If there exists a permuting tri-derivation $D: M \times M \times M \rightarrow$ $M$ such that dis skew-commuting on $I$, where $d$ is the trace of $D$, then $d$ is commuting on $I$.

\section{Proof:}

Using the same method in Theorem 2.7, with Lemma 1.7, we complete the proof of the Theorem. 


\section{Theorem 2.9}

Let $M$ be a non commutative 3-torsion free semiprime $\Gamma$-ring satisfying the condition (*) and $I$ be a nonzero ideal of $M$. If there exists a permuting tri-derivation $D: M \times M \times M \rightarrow$ $M$ such that $d$ is skew- centralizing on $I$ where $d$ is the trace of $D$, then $d$ is commuting on $I$.

\section{Proof:}

Using same method in Theorem 2.7, we obtain $[d(x) \alpha y \delta y, r]_{\beta} \in Z(M)$ for all $x \in I, r \in M, \alpha$, $\beta, \delta \in \Gamma$, replacing $r$ by $y$ with using Lemma 1.7, we complete the proof of the theorem.

\section{Corollary 2.10}

Let $M$ be a 3-torsion free prime $\Gamma$-ring satisfying the condition $\left(^{*}\right)$ and $I$ be a nonzero ideal of $M$. If there exists a nonzero a permuting tri-derivation $D: M \times M \times M \rightarrow M$ such that $d$ is skew-centralizing on $I$ where $d$ is the trace of $D$, then $M$ is commutative.

\section{Proof:}

Suppose that $M$ is non-commutative, then by the same method in Theorem 2.9, we get $[d(x), \mathrm{x}]_{\beta} \in Z(M)$ for all $x \in I, \beta \in \Gamma$. Hence by Lemma 1.8 , the proof of the corollary is complete.

\section{References}

1. N. Nabusawa, Osaka J. Math. , 65 (1964).

2. W. E. Barnes, Pacific J. Math 18, 411 (1966).

3. M. A. Ozturk, M. Sapanci, M. Soyturk and K. H. Kim, Sci. Math. Jpn. 53, 3491 (2001).

4. M. A. Ozturk, East Asian Math. J. 15, 2177 (1999).

5. M. A. Ozturk, M. Sapanci and Y. B. Jun, East Asian Math. J. 15, 1105 (1999).

6. Y. -S. Jung and K.-H. Park, Bull. Korean Math. Soc., 44789 (2007). http://dx.doi.org/10.4134/BKMS.2007.44.4.789

7. M. A. Ozturk and Young Bae Jun, Commun. Fac. Sci. Univ. Ank. Series A1 54, 11 (2005).

8. D. Ozden and M. A. Ozturk, Kyungpook Math. J. 46, 153 (2006).

9. M. A. Ozturk and M. Sapanci, Hacettepe Bul. of Sci. and Engineering, Series B 26, 31 (1997).

10. J. Vukman, Aequationes Math. 38 (2-3), 245 (1989). http://dx.doi.org/10.1007/BF01840009

11. J. Vukman, Aequationes Math. 40 (2-3), 181 (1990). http://dx.doi.org/10.1007/BF02112294

12. K. K. Dey and A. C. Paul, J. Sci. Res. 4 (1), 33 (2012). http://dx.doi.org/10.3329/jsr.v4i1.7911

13. K. K. Dey and A. C. Paul, J. Sci. Res. 3 (2), 331 (2011). http://dx.doi.org/10.3329/jsr.v3i2.7278

14. K. K. Dey and A. C. Paul, J. Sci. Res. 4 (2), 349 (2012). http://dx.doi.org/10.3329/jsr.v4i2.8691

15. K. K. Dey, A. C. Paul and I. S. Rakhimov, JP Journal of Algebra, Number Theory and Applications 25 (1), 29 (2012). 